\title{
The natural history of pre-Type 1 (insulin-dependent) diabetes mellitus in patients with autoimmune endocrine diseases
}

\author{
C. Betterle $^{1}$, F. Presotto ${ }^{1}$, L.Magrin ${ }^{1}$, B.Pedini ${ }^{1}$, L. Moro ${ }^{2}$, A. Caretto ${ }^{1}$, R.Zanchetta ${ }^{1}$ \\ ${ }^{1}$ Istituto di Semeiotica Medica, University of Padua, Padua, Italy \\ ${ }^{2}$ General Hospital of Treviso, Treviso, Italy
}

\begin{abstract}
Summary An 11-year prospective study was carried out in 180 non-diabetic patients with organ-specific autoimmune diseases to evaluate islet cell antibodies in predicting Type 1 (insulin-dependent) diabetes mellitus. Islet cell antibodies were characterised according to titres, persistence, complement-fixing ability, and pattern. During follow-up, 14 of 46 patients with islet cell antibodies persistently greater than 5 Juvenile Diabetes Foundation Units (JDF-U) (30.4\%), none of 23 with islet cell antibodies between 2.5 and 5 JDF-U or fluctuating, and 3 of 109 without islet cell antibodies $(2.7 \%)$, developed diabetes. The cumulative risk of developing diabetes was $70 \%, 0 \%$, and $4 \%$, respectively. All the patients who developed diabetes were females. Eight progressed to insulin-dependence acutely, four showed a transient period of non-insulin-dependence, while two were still insulin-free. No difference was found in titres of islet cell antibodies for the risk of diabetes. Comple-
\end{abstract}

ment-fixing islet cell antibodies enhanced the cumulative risk for the disease in patients with conventional islet cell antibodies at low-middle ( $\geq 2.5-40 \mathrm{JDF}-\mathrm{U})$, but not at high ( $\geq 80 \mathrm{JDF}-\mathrm{U})$ titres. Forty-two patients with islet cell antibodies were investigated for the whole or the selective pattern. In the presence of the whole pattern the cumulative risk for diabetes rose to $100 \%$, while with the selective pattern it declined to $34 \%$. The whole pattern was found in $83 \%$ of patients who developed Type 1 diabetes acutely. In patients with organ-specific autoimmune diseases, the whole islet cell antibody pattern greatly enhances the prediction for diabetes. [Diabetologia (1994) 37:95-103]

Key words Autoimmune disease, Type 1 (insulin-dependent) diabetes mellitus, islet cell antibodies, autoimmune polyendocrinopathy, HLA-DR.
Type 1 (insulin-dependent) diabetes mellitus is considered an organ-specific autoimmune disease affecting individuals with a genetic susceptibility $[1,2]$. It has also been observed that Type 1 diabetes is associated with other endocrine autoimmune disorders, mainly thyro-gastric and adrenal, more frequently than would be expected in a background population [3, 4]. Similar to other organ-specific autoimmune diseases, where the relevant circulating autoantibodies may be found many years before they become manifest $[5,6]$, Type 1 diabetes has a long silent prodromal period. In geneti-

Received: 26 April 1993

and in revised form: 3 August 1993

Corresponding author: Professor C. Betterle, Istituto di Semeiotica Medica, Chair of Allergology and Clinical Immunology, Via Ospedale, 105, I-35 128 Padova, Italy cally susceptible individuals, before the clinical onset of the disease the immune system begins to produce antibodies against a variety of pancreatic islet cell autoantigens [7]. Islet cell antibodies (ICA), originally identified in patients with autoimmune polyglandular failure [8], have been extensively investigated, and their detection has become common in most laboratories. Furthermore, efforts have made to standardise their measurement $[9,10]$. Previous investigations have shown that ICA, especially when fixing complement or at high titres, confer elevated risk for developing diabetes in unaffected individuals, such as in identical twins and triplets [11, 12], first-degree relatives (FDR) [13-16], schoolchildren [17-19], and patients with organ-specific autoimmunity $[20,21]$. Although ICA have been reported to be strong markers of potential diabetes, many ICA-positive subjects remain disease-free after 


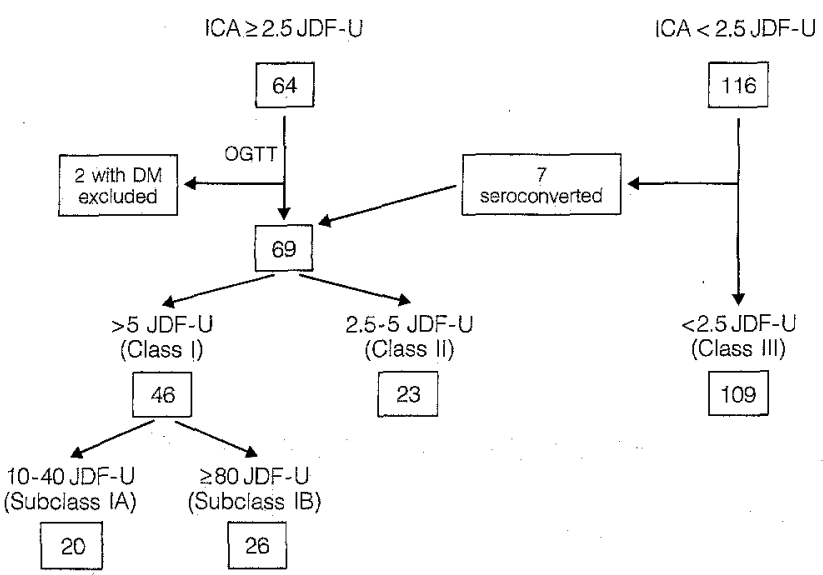

Fig. 1. Organ-specific autoimmune patients with different classes of islet cell antibodies (ICA) who entered into the study. DM, diabetes mellitus; OGTT, oral glucose tolerance test; JDF$\mathrm{U}$, Juvenile Diabetes Foundation Units

several years of follow-up. It has recently been shown that ICA are heterogeneous $[22,23]$, and this may account for the different rates of progression to diabetes reported in susceptible individuals $[22,23]$.

In this study, 180 non-diabetic patients with organspecific autoimmune diseases were followed-up for a maximum of 11 years to investigate the role of ICA according to their titres, behaviour, complement-fixing ability, and pattern, as serological markers in predicting Type 1 diabetes. Sex, genetics, and other factors have been also considered.

\section{Subjects and methods}

\section{Subjects}

Between September 1976 and December 1991 autoantibody screening was performed on 3,042 patients with one or more organ-specific autoimmune diseases (OSAD), but without overt diabetes (mean age 40.1 years, range 5-74;2,541 females and 501 males, female/male ratio $=5.1$ ). All were living in north-eastern Italy, mostly in the Veneto region. Patients with ascertained primary endocrine deficiencies were receiving hormone replacement therapy (1-thyroxine and/or cortisone acetate), those with active Graves' disease were treated with antithyroid drugs.

Of those patients with ICA of 2.5 Juvenile Diabetes Foundation Units (JDF-U) or more, 64 were enrolled for the prospective survey (Fig. 1). They were investigated for ICA-IgG titres and complement-fixing (CF)-ICA. ICA-positive patients were re-evaluated for ICA-IgG, ICA titres, and CF-ICA every 214 months (mean 7 months). The mean duration of their followup was 43.1 months (range 2-132), for a total observation period of 2,974 months. The average number of serum determinations was 5.4 per patient (range 2-12), for a total of 346 tests. Fortytwo subjects with persistent ICA of more than 5 JDF-U have also been retrospectively investigated to define their ICA pattern.

Another 116 randomly selected OSAD patients without detectable ICA ( $<2.5 \mathrm{JDF}-\mathrm{U})$ were also followed-up. They were subsequently investigated by repeated tests for ICA and basal glycaemia every $12-36$ months (mean 22 months). The mean time of their follow-up was 52.1 months (range 2-144), for a total observation period of 5,675 months. The average number of sera samples tested was 2.2 per patient, for a total of 255 determinations. All the 180 subjects were questioned concerning family history of diabetes. Prior informed consent was obtained from all patients where appropriate. The investigation was performed in accordance with the principles of the Declaration of Helsinki.

A further 180 unselected patients with newly-diagnosed Type 1 diabetes were also interviewed to establish family history of diabetes, and to compare it with that of OSAD patients.

\section{Islet cell antibodies of the IgG class (ICA-IgG)}

ICA-IgG were determined using the standard indirect immunofluorescence technique, according to the protocol proposed by the International Committee on Cytoplasmic Islet cell Antibody Standardisation $[9,10]$.

All 3,042 sera were tested undiluted, at $1 / 2$ and $1 / 4$ dilution in the same session. If the positivity exceeded $1 / 4$, sera were further tested at $1 / 8,1 / 16,1 / 32$, and so on, up to the end-point. ICA titres were therefore converted to JDF-U by comparison with the standard curve, obtained by diluting the Reference International Standard Serum supplied by the Immunology and Diabetes Workshops (IDW) Committee, which had a titre of 80 JDF-U. Accordingly, sera with ICA titres 1/1, 1/2, 1/4, 1/8,1/16, $1 / 32$, and more than $1 / 32$ were regarded as having values of 2.5 , $5,10,20,40,80$, and more than $80 \mathrm{JDF}-\mathrm{U}$, respectively. Undiluted sera that gave negative reactions were conventionally considered to have ICA less than 2.5 JDF-U. In our laboratory, 54 of the 57 negative control sera, supplied blindly in 1991 by the IDW Committee, were found to have ICA values less than 2.5 JDF-U, while 3 were found ICA positive with a titre of 5 JDF-U. In the periodical Proficiency Tests performed from 1988 to 1991 , our laboratory revealed a validity ranging from 93 to $100 \%$, a consistency from 87 to $100 \%$, a sensitivity from 81 to $100 \%$, and a specificity of $100 \%$.

\section{Complement-fixing (CF) ICA}

In the 69 ICA-positive patients followed-up, CF-ICA were detected by standard indirect immunofluorescence technique on normal human pancreas of blood group 0 . Normal human serum was used as the source of complement followed by rabbit antihuman serum $\mathrm{C} 3$ fluorescein isothiocyanate-conjugated (FITC) (Behringwerke, Marburg, Germany), as previously described [20].

\section{Classes of ICA}

According to titres and behaviour of ICA during follow-up, patients were arbitrarily ranked into three classes.

Class $I$ included patients who, during follow-up, had ICA values mainly 5 JDF-U or more, or who persistently seroconverted for ICA beyond this level. This class was further split in two subclasses, if ICA were detectable mainly at middle (10-40 JDF-U, Subclass IA) or high ( $\geq 80$ JDF-U, Subclass $I B$ ) titres.

Class II included patients with ICA mainly between 2.5 and 5 JDF-U. Fluctuating ICA were considered as those with values moving repeatedly above and below the threshold of $2.5 \mathrm{JDF}-\mathrm{U}$.

Class III included patients who permanently remained ICAnegative ( $<2.5$ JDF-U). Patients who developed a seropositivity for ICA during follow-up, i. e. those moving from ICA values less than 2.5 JDF-U to more than $5 \mathrm{JDF}-\mathrm{U}$, and who persistently held such a positivity, were transferred from ICA Class III into Class $I$. 


\section{ICA pattern}

Forty-two patients with persistent ICA of 5 JDF-U or more were further investigated to identify their ICA pattern by a four-layer immunofluorescence technique according to Genovese et al. [22]. All serum samples were retested by the same technique after incubation with rat brain homogenates [22].

\section{Metabolic study}

ICA-positive patients underwent an oral glucose tolerance test (OGTT) at the start of the study, and periodically during ICA evaluations, by plasma glucose assessment at 0 and $120 \mathrm{~min}$, according to the criteria of the National Diabetes Data Group [24]. ICA-negative patients underwent OGTT at the beginning of their investigation, and were retested for basal glycaemia at the time of their ICA determination.

\section{Genetic study}

Fifty-nine OSAD patients, 35 ICA-positive and 24 ICA-negative, were typed for HLA-DR locus by a standard microlymphocytotoxicity technique.

\section{Statistical analysis}

Differences between the various groups and subgroups of subjects were evaluated by contingency tables (chi-square test) with Yates' correction for continuity if the number in any expected class was five or less. Fisher's exact test was employed if any class was zero. Actuarial survival rates, according to the CutlerEderer method [25], were adopted to estimate the likelihood of progression toward diabetes. All patients entered the life-table when ICA were first investigated in our laboratory. The followup ended when diabetes developed, which was referred to as the event of interest; or when ICA were last detected for nondiabetic individuals. The results of survival analysis were plotted drawing curves of "cumulative risk of morbidity" (CR), at 11 years, regarding ICA status. The log-rank statistic was used to compare the estimates between the selected categories [26]. If two groups did not show the same length of observation time when compared, the significance level was calculated at the last time-interval common to both the groups considered. The nonparametric Mann-Whitney U test was additionally employed to evaluate differences between curves, and a two-tailed $p$ value of 0.05 was considered to indicate statistical significance. The annual incidence of diabetes in the cohorts of interest was calculated by dividing the number of diabetic patients by the patient years of follow-up. Each patient contributed to the sum of patient years a period (in months) from the beginning of their observation until diabetes was diagnosed or the observation ended. Sampling errors and $95 \%$ confidence intervals (CI) were calculated.

\section{Results}

\section{$I C A$ in $O S A D$ patients}

Of 3,042 subjects with OSAD, $110(3.6 \%)$ had detectable ICA ( $\geq 2.5$ JDF-U) in their sera. OGTT, initially performed on 64 ICA-positive non-selected patients, revealed a normal glucose tolerance (NGT) in 57, an impaired glucose tolerance (IGT) in 3 , a previous abnormal glucose tolerance (Prev-AGT) in 2, and diabetes in 2 individuals who were then excluded from the study. The 7 patients who seroconverted during follow-up, and arising from Class III, were added to the initial 62 cases. So, the final group with persistent ICA included 69 subjects (Fig. 1).

Fifty-eight were females and 11 males (female/male ratio $(F / M)=5.3$ ), with a mean age of 40.6 years (range 6-71) (22 with Graves' disease, 3 with Graves' disease and vitiligo, 2 with Graves' disease and type A chronic atrophic gastritis, 25 with Hashimoto's thyroiditis or idiopathic myxoedema, 1 with Hashimoto's thyroiditis and type A chronic atrophic gastritis, 5 with vitiligo, 3 with type I autoimmune polyendocrine syndrome (APS), 3 with type II APS, 2 with idiopathic Addison's disease, 2 with alopecia areata, and 1 with type A chronic atrophic gastritis).

In all the 116 OSAD patients without ICA (i.e., $<2.5$ JDF-U), the OGTT initially performed showed NGT. During periodic testing, 7 patients became ICA positive, and from then were included into Class $I$ of ICA. Seroconversion was documented after a mean observation period of 25.2 months (range 3-44). Therefore, the persistently ICA-negative group included 109 OSAD patients (Fig. 1). Ninety-two were females and 17 males $(F / M=5.4)$, with a mean age of 40 years (range 9-70) (55 with Graves' disease, 25 with Hashimoto's thyroiditis or idiopathic myxoedema, 8 with Addison's disease or with adrenal autoantibodies but without overt hypoadrenalism, 12 with type II APS, 3 with type I APS, 4 with vitiligo, and 2 with alopecia areata).

\section{Classes of ICA, fluctuating ICA, CF-ICA}

Of the 69 OSAD patients with ICA of $2.5 \mathrm{JDF}-\mathrm{U}$ or more, $46(66.7 \%)$ belonged to Class $I$, and $23(33.3 \%)$ to Class II.

Of the 46 Class I patients, 20 belonged to Subclass $I A$, and 26 to Subclass IB. Of the 20 Subclass $I A$ patients, 2 showed fluctuation of ICA, $3(15 \%)$ were persistently positive for CF-ICA, $4(20 \%)$ were occasionally CF-ICA positive, and $13(65 \%)$ were found to be repeatedly negative. Of the 26 Subclass $I B$ patients, none showed ICA fluctuation, $22(84.6 \%)$ had persistent CF-ICA, 3 (11.5\%) had occasional CF-ICA, and 1 $(3.8 \%)$ was persistently negative (Table 1 ).

Of the 23 Class II patients, 14 revealed fluctuation of ICA, and none had CF-ICA.

None of the 109 Class III patients had CF-ICA.

A significant association was found between persistent high titres of ICA-IgG (Subclass $I B$ ) and persistence of CF-ICA $\left(p=0.001 \times 10^{-2}\right.$, chi-square test $)$. 
Table 1. Classes of ICA in OSAD patients: relationship with CF-ICA and progression to diabetes

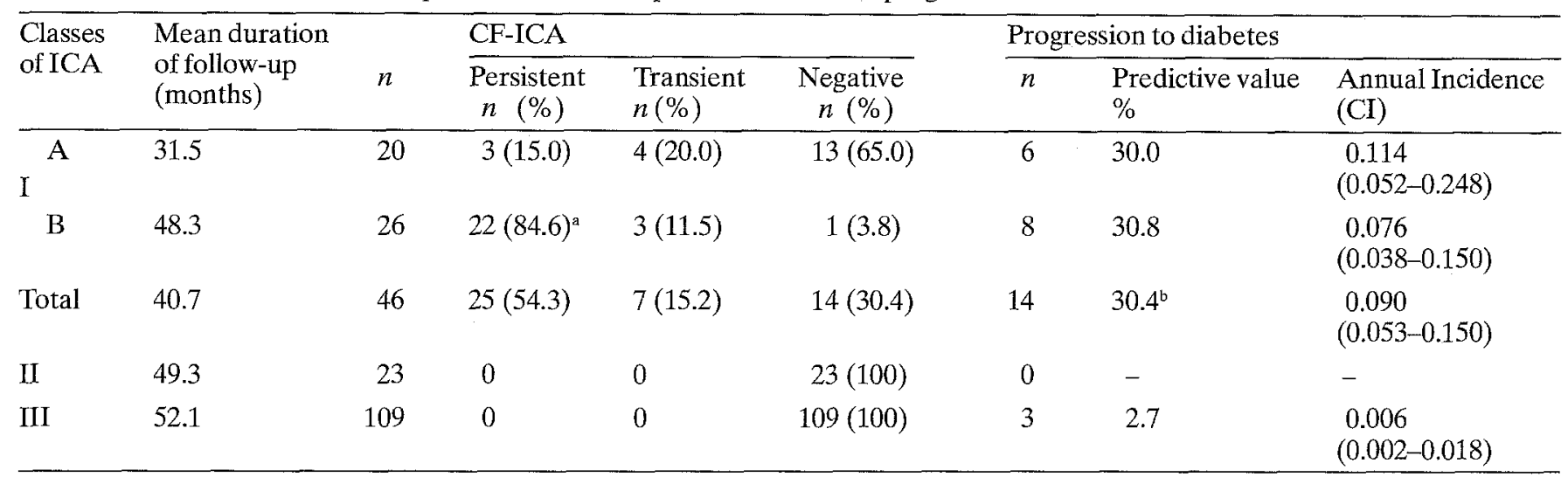

${ }^{\mathrm{a}} p<0.001$ vs Subclass IA; ${ }^{b} p=0.008$ vs Class II and $<0.0001$ vs Class III.

OSAD, organ-specific autoimmune disease; ICA, islet cell antibodies; CF, complement-fixing; CI, confidence interval; Class I,
ICA persistently > 5 JDF-U; Subclass IA, ICA 10-40 JDF-U; Subclass IB, ICA $\geq 80 \mathrm{JDF}-\mathrm{U}$; Class II, ICA 2.5-5 JDF-U or fluctuating; Class III, ICA persistently $<2.5$ JDF-U

Table 2. CF-ICA and progression to diabetes in OSAD patients

\begin{tabular}{|c|c|c|c|c|c|c|}
\hline \multirow[b]{2}{*}{ Classes of ICA } & \multirow[b]{2}{*}{ CF-ICA status } & \multirow[b]{2}{*}{$\begin{array}{l}\text { Mean duration of } \\
\text { follow-up (months) }\end{array}$} & \multirow[b]{2}{*}{$n$} & \multicolumn{3}{|c|}{ Progression to diabetes } \\
\hline & & & & $n$ & $\begin{array}{l}\text { Predictive value } \\
\%\end{array}$ & $\begin{array}{l}\text { Annual Incidence } \\
\text { (CI) }\end{array}$ \\
\hline $\mathrm{I}(\mathrm{A}+\mathrm{B})$ & CF-ICA T/N & 31.0 & 21 & 4 & 19.0 & $\begin{array}{l}0.073 \\
(0.028-0.189)\end{array}$ \\
\hline \multirow{2}{*}{ II } & CF-ICA PP & - & - & 0 & - & - \\
\hline & CF-ICA T/N & 49.3 & 23 & 0 & - & - \\
\hline III & - & 52.1 & 109 & 3 & 2.7 & $\begin{array}{l}0.006 \\
(0.002-0.018)\end{array}$ \\
\hline
\end{tabular}

CF-ICA, complement-fixing islet cell antibodies; $\mathrm{PP}$, persistently positive; $\mathrm{T}$, transiently positive; $\mathrm{N}$, persistently negative

\section{ICA pattern}

Of the 42 subjects evaluated, 17 showed the whole and 25 the selective pattern of ICA. All sera with the whole pattern maintained the same reaction after absorption with rat brain homogenate, while all sera with the selective pattern lost this reactivity. The whole pattern was found in $82 \%$ of patients with persistently high ICA titres (Subclass $I B)$, and in $76 \%$ of those with persistent CF-ICA. These associations, however, were not statistically significant ( $p=0.09$ and $p=0.20$, respectively).

\section{Classes of ICA and progression to diabetes (Table 1)}

During follow-up 17 patients with OSAD developed diabetes. Of the 46 Class $I$ patients, $14(30.4 \%)$ developed diabetes, with a mean latency period of 36.6 months (range 2-129) (annual incidence $(\mathrm{AI})=$
0.090; $\mathrm{CR}=70 \%$, CI 56-84) (Table 1, Fig. 2 a). In particular, diabetes occurred in 6 patients $(30 \%)$ of $S u b$ class $I A$ ( $\mathrm{AI}=0.114 ; \mathrm{CR}=76 \%, \mathrm{CI} 38-114)$, and in 8 $(30.8 \%)$ of Subclass IB (AI $=0.076 ; \mathrm{CR}=62 \%$, CI $48-$ 76) (Table 1, Fig. 2b). No significant difference was shown in the cumulative risk for diabetes between $S u b$ class $I A$ and $I B$. In general, the ICA titres in patients developing the disease did not vary more than two dilutions with respect to the initial antibody detection. After the onset of diabetes, ten patients were further followed-up for a mean period of 3.5 years. Five patients became ICA-negative, three showed a decrease in ICA titres, while two maintained the same ICA trend. None of the 23 Class $I I$ patients developed diabetes. Of the 109 patients belonging to Class III , 3 $(2.7 \%)$ developed diabetes after a mean observation period of 11.7 months (range 7-15) $(\mathrm{AI}=0.006$; $\mathrm{CR}=4 \%, \mathrm{CI} 0-8)$. The cumulative risk for diabetes in Class I patients was significantly increased if compared 

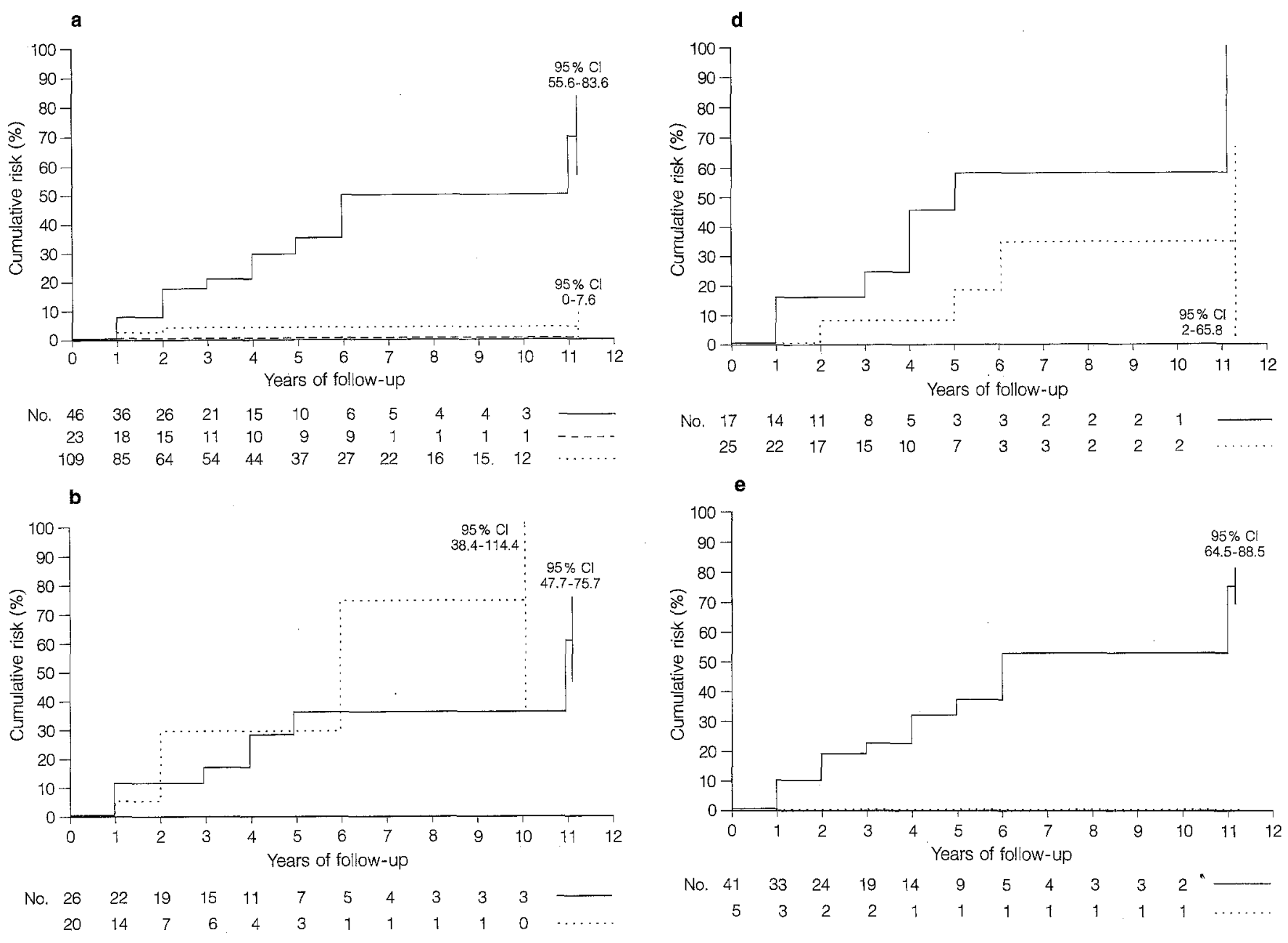

No. $\begin{array}{rrrrrrrrrrr}41 & 33 & 24 & 19 & 14 & 9 & 5 & 4 & 3 & 3 & 2 \\ 5 & 3 & 2 & 2 & 1 & 1 & 1 & 1 & 1 & 1 & 1\end{array}$

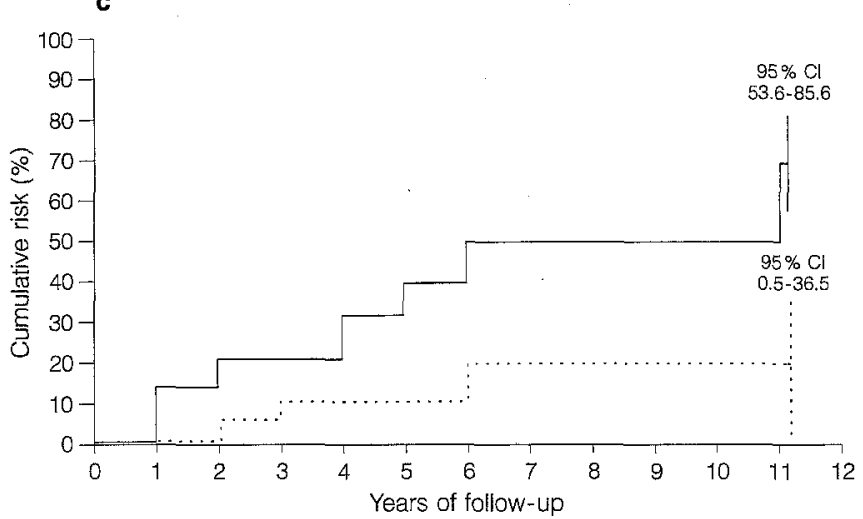

No. $\begin{array}{rrrrrrrrrrrr}25 & 20 & 17 & 15 & 11 & 7 & 4 & 4 & 3 & 3 & 3 & \\ 44 & 34 & 24 & 17 & 14 & 12 & 11 & 2 & 2 & 2 & 1 & \ldots \ldots\end{array}$

Fig.2a-e. Estimated probability of progression toward diabetes according to (a) islet cell antibody (ICA) Classes (Class I-, Class

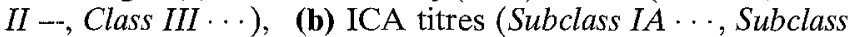
$I B-$ ), (c) complement-fixing (CF)-ICA (CF-ICA positive-, CFICA negative..$)$, (d) ICA pattern (whole-, selective $\cdots$ ), and (e) sex (ICA-positive females -, ICA-positive males $\cdots$... The cumulative morbidity rate for diabetes was significantly increased in patients with ICA persistently $>5 \mathrm{JDF}-\mathrm{U}$ (Class $I$ ) vs Class $I I(p=0.005$, log-rank test) and ICA-negative (Class III) $\left(p=0.004 \times 10^{-4}, \log\right.$-rank test) (a), with CF-ICA persistently positive vs CF-ICA negative ( $p=0.002$, log-rank test) (c), with whole vs selective pattern of ICA ( $p=0.02$, log-rank test) (d), and in females vs males $(p<0.0001$, U-test) (e). No significant difference was found between patients with ICA $\geq 80 \mathrm{JDF}-\mathrm{U}$ (Subclass IB) and 10-40 JDF-U (Subclass IA) (b)

both to Class II $(p=0.005)$ and Class III ( $p$ $=0.004 \times 10^{-4}$, log-rank test) (Table 1, Fig. $2 \mathrm{a}$ ).

\section{CF-ICA and progression to diabetes (Table 2)}

According to CF-ICA status within Class I, the onset of diabetes was found in 10 of $25(40 \%)$ patients with persistent CF-ICA $(\mathrm{AI}=0.098 ; \mathrm{CR}=70 \%$, CI 54-86),

compared to 4 of $21(19 \%)$ with transient or negative CF-ICA (AI $=0.073 ; \mathrm{CR}=50 \%$, CI 6-94). The risk for diabetes in patients with persistent CF-ICA was significantly higher than that of patients without or with occasional CF-ICA belonging to Class $I$ and $I I$ (i.e. with ICA $\geq 2.5 \mathrm{JDF}-\mathrm{U}$ or fluctuating) $(\mathrm{AI}=0.026 ; \mathrm{CR}=$ $18.5 \%$, CI $0.5-36.5)\left(\chi^{2}=5.086, p=0.02, \log\right.$-rank test $)$ (Fig. 2c). In Subclass IA, diabetes occurred in 3 of 3 $(100 \%)$ patients with persistent $\mathrm{CF}-\mathrm{ICA}(\mathrm{AI}=0.363$, 
CI $0.122-1.019 ; \mathrm{CR}=100 \%)$, and in 3 of $17(18 \%)$ with transient or negative CF-ICA (AI $=0.067$, CI $0.023-$ $0.197 ; \mathrm{CR}=60 \%$, CI 31-89) $\left(\chi^{2}=3.12, p=\mathrm{NS}, \log -\right.$ rank test; $p=0.006$, U-test). Within Subclass $I B$, diabetes developed in 7 of $22(32 \%)$ patients with persistent CF-ICA $(\mathrm{AI}=0.075, \mathrm{CI} 0.036-0.156 ; \mathrm{CR}=$ $61 \%$, CI $47-75)$, compared to 1 of 4 (25\%) with transient or negative CF-ICA (AI $=0.081$, CI 0.014-0.459; $\mathrm{CR}=40 \%, \mathrm{CI} 9-71)(p=\mathrm{NS}, \log$-rank and U-test $)$.

\section{ICA pattern and progression to diabetes}

Of the 42 patients evaluated, 12 developed diabetes and 30 had NGT.

Of the 12 patients who developed diabetes, $8(67 \%)$ showed the whole, and $4(33 \%)$ the selective ICA pattern. Of the 30 patients who did not develop diabetes, 9 $(30 \%)$ had the whole and $21(70 \%)$ the selective pattern. Thus, diabetes occurred in 8 of $17(47 \%)$ patients with the whole pattern ( $\mathrm{AI}=0.132$, CI $0.067-0.261$; $\mathrm{CR}=100 \%)$, compared to 4 of $25(16 \%)$ patients with the selective pattern $(\mathrm{AI}=0.042, \mathrm{CI} 0.016-0.107$; $\mathrm{CR}=$ $34 \%$, CI 2-66) $\left(\chi^{2}=4.710, p=0.02\right.$, log-rank test $)$ (Fig. 2d).

Six patients developed Type 1 diabetes with acute onset, while six had a slower onset. The whole pattern was found in five of the six $(83 \%)$ patients in whom diabetes presented acutely, with respect to three of the six $(50 \%)$ in whom diabetes presented as non-insulin requiring.

\section{Sex and progression to diabetes}

Of the 46 Class I patients, 41 were females and 5 males. All 14 patients who developed diabetes were females ( $p<0.0001$, U-test) (Fig. 2e). Of 109 Class III patients, 92 were females and 17 males. The 3 patients who developed diabetes were females.

\section{Family history of Type 1 diabetes in OSAD patients and in the patients with Type 1 diabetes}

A family history for Type 1 diabetes was found in six (3\%), and for Type 2 (non-insulin-dependent) diabetes in nine $(5 \%)$ of our OSAD patients.

Of the 180 patients with newly-diagnosed Type 1 diabetes, $17(9 \%)$ were found to have one or more first degree relatives with Type 1 and $10(5.5 \%)$ with Type 2 diabetes. One diabetic patient had a family history of both Type 1 and Type 2 diabetes $(0.5 \%)$.

\section{$H L A-D R$ in OSAD patients}

Of 59 OSAD patients typed for HLA, $31(52.5 \%)$ had DR3 and/or DR4 (17 DR3, 6 DR4, and 8 DR3/DR4). Specifically, 18 of 35 patients $(51.4 \%)$ with ICA had DR3 and/or DR4 (9 DR3, 3 DR4, and 6 DR3/DR4), while 17 had other DR haplotypes. Thirteen of 24 patients $(54.2 \%)$ without ICA had DR3 and/or DR4 (8 DR3, 3 DR4, and 2 DR3/DR4), while 11 had other haplotypes. No differences were observed in the prevalence of DR3 and/or DR4 between OSAD patients with or without ICA, whereas the prevalence of both DR3 and DR4 in OSAD patients was significantly increased with respect to the background population (DR3 $=19 \%$, and DR4 $=6 \%$ in 134 normal control subjects) ( $p=0.0007$ and $p=0.0005$, respectively).

\section{HLA-DR and progression to diabetes}

Fifteen of the 17 patients who developed diabetes were typed for HLA-DR, $10(67 \%)$ having DR3 and/or DR4 (5 DR3, 3 DR4, and 2 DR3/DR4), and 5 showing other DR haplotypes. Of 44 patients investigated for HLADR who did not progress to diabetes, $21(48 \%)$ had DR3 and/or DR4 (12 DR3, 3 DR4, and 6 DR3/DR4), while the remaining patients had other haplotypes. Despite the fact that two thirds of the patients who later developed diabetes had DR3 and/or DR4, no statistical difference was found when they were compared to OSAD patients who had not developed the disease.

\section{HLA-DR and ICA pattern}

Twenty-three patients with persistent ICA were evaluated for both ICA pattern and HLA-DR haplotype. Of 11 patients with the whole pattern, 7 (64\%) had DR3 and/or DR4, and 4 other haplotypes (none was DR2). Of 12 patients with the selective pattern, $4(33 \%)$ had DR3 and/or DR4, while 8 had other haplotypes, 3 of which $(25 \%)$ were DR2. Thus, DR3 and/or DR4 showed an increased frequency in sera with the whole pattern, although this was not statistically significant $(p=0.30)$.

\section{Clinical features of patients who developed diabetes (Table 3)}

Of the 69 ICA-positive patients, 14 progressed to diabetes. Upon entry to the study, 10 had NGT, 3 IGT, and 1 Prev-AGT, and all were females. Thus, the prevalence of diabetes in females was $34 \%$ compared to $0 \%$ in males. Three belonged to the group of the seven subjects who had seroconverted for ICA, so that the prevalence of diabetes in such individuals was $43 \%$. Diabetes occurred after a mean observation period of 
Table 3. Immunologic, genetic and metabolic features in ICA-positive (patients 1-14) and ICA-negative (patients 15-17) OSAD patients who developed diabetes during follow-up

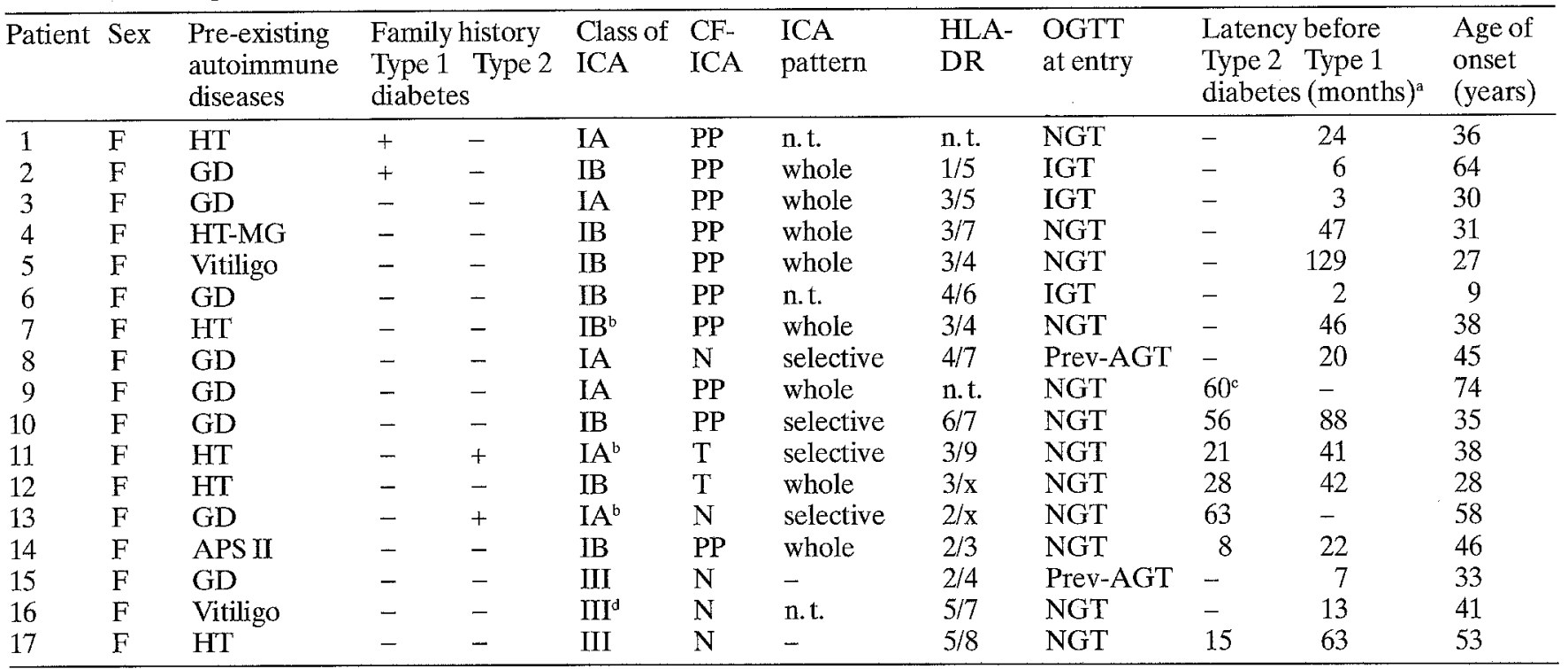

${ }^{a}$ Period of observation before the onset of diabetes.

${ }^{b}$ Seroconverted for ICA before the onset Type 1 diabetes.

'Deceased.

${ }^{d}$ Seroconverted for ICA at the onset of Type 1 diabetes.

HLA, Human leucocyte antigens; OGTT, oral glucose tolerance test; HT, Hashimoto's thyroiditis; GD, Graves' disease; MG, my-

36.6 months (range $2-129$ ) at a mean age of 40.3 years (range 9-74). Two patients had a family history of Type 1 diabetes, and two of Type 2 diabetes. In eight patients the disease presented acutely as insulin-dependent. The onset was non-acute in six individuals: four required insulin after 14-32 months of therapy with oral hypoglycaemic agents (OHA) and/or diet (patients no. 10,11,12,14), one is still well-controlled by OHA after 15 months from diagnosis (patient no. 13 ), while one patient died of myocardial infarction after 20 months from the onset of the disease (patient no. 9). In the three seroconverted patients who developed diabetes (patients no. 7,11,13), the disease developed after a mean period of 43.3 months (range 2163) from seroconversion.

Of the 109 OSAD patients without ICA, three progressed to diabetes after a mean observation period of 11.7 months (range 7-15) (patients no. 15, 16, 17), all were females. In one, the disease appeared as non-insulin-dependent (patient no. 17), and in two as insulindependent (patients no. 15, 16). Patient no. 17 required insulin therapy after 48 months. None had a family history of Type 1 or Type 2 diabetes. Patient no. 16 was found to be ICA positive at the onset of diabetes, having shown a negative ICA test 1 year before.

Of the remaining 32 patients belonging to Class $I$, all maintained NGT during follow-up. One 17-year-old male with type I autoimmune polyendocrine syndrome, with persistently high titres of ICA, CF-ICA, selective ICA-pattern, and DR5/DR7 HLA-haplotype, asthenia gravis; APS II, type II autoimmune polyglandular syndrome; AD, Addison's disease; $P$, persistently positive; T, transiently positive; $\mathrm{N}$, persistently negative; NGT, normal glucose tolerance; IGT, impaired glucose tolerance; Prev-AGT, previous abnormal glucose tolerance; n.t., not tested

died after 121 months of follow-up from cerebral haemorrhage during a hypertensive crisis. He was also suffering from renal insufficiency. Histological investigation of his pancreas did not show any sign of lymphocytic insulitis [27].

\section{Discussion}

Over the past 10 years many prospective studies have been carried out on selected populations at risk for diabetes in order to characterise and, possibly, quantify immunological and genetic factors conferring susceptibility. Investigation of patients with autoimmune endocrinopathies is important because of the polyglandular involvement in their disease. However, only few studies are available thus far $[20,21]$. The present investigation, which follows the recent "polyendocrine study" carried out in southern England [21], reports more extensive data about a prospective Italian study which began in 1976.

During screening, $3.6 \%$ of patients with OSAD had ICA of 2.5 JDF-U or more, and about two-thirds of them showed persistent ICA levels of more than 5 JDF-U. A group of patients showed fluctuations in ICA status, but they belonged almost exclusively to those with the lowest ICA titres. Thus, fluctuation seems more related to methodological variability than to actual modifications in ICA amounts. High ICA titres were strictly correlated with the ability to fix complement, confirming that 
CF-ICA are likely to be the expression of high levels of ICA-IgG. After 11 years of follow-up, the predictive value for diabetes in OSAD patients having ICA persistently greater than $5 \mathrm{JDF}-\mathrm{U}$ was $30 \%$, with an estimated actuarial risk of $70 \%$. In the polyendocrine study, Bosi and colleagues [21] found a positive predictive value of $20 \%$, with a cumulative risk of $41 \%$ after 10 years. Our elevated actuarial estimate at 11 years may be partly due to the smaller number of patients available for complete evaluation over the last years of follow-up, and to the bias introduced by the assumption that the future experiences of subjects lost to follow-up are similar to those remaining under observation. However, both the studies suggest that OSAD patients are generally less prone to develop diabetes if compared to FDR of Type 1 diabetic probands, where the predictive value ranges from 25 to $75 \%[14,15,28]$, or to identical twins, where the predictive value rises to $77 \%$ [12]. Such differences for the development of diabetes in FDR have been correlated to HLA identity or haploidentity with the diabetic proband [13].

The lower progression toward diabetes in our OSAD patients compared to FDR may be explained by the fact that a family history of Type 1 diabetes was ascertained in only $3 \%$. This concept is supported by the observation that ICA-positive OSAD patients with a family history of Type 1 diabetes develop the disease at a similar rate to ICA-positive FDR [21]. These findings also suggest that, in one individual with ICA, a family history of Type 1 diabetes confers more risk for the future onset of the disease than the presence of organspecific autoimmunity. If this is the case, the coincidence of OSAD and family history of Type 1 diabetes in one person would not provide more risk to develop diabetes than in one FDR without OSAD.

Considering three different studies carried out on the general population (schoolchildren), the cumulative predictive value of ICA has been estimated to be about $10 \%$ [17-19]. Thus, ICA confer a declining risk for diabetes in identical twins, FDR, OSAD patients, and schoolchildren, respectively.

We did not find a significant difference in the risk for diabetes between middle and high ICA titre in our patients. These data agree with those recently reported by Bosi et al. [21], but they differ substantially from that observed in FDR, where ICA levels are proportionally related to the risk of progression to diabetes $[15,28]$.

A positive test for CF-ICA was an additional predictor of Type 1 diabetes if the ICA titres were generally 2.5 JDF-U or more, but not if they were persistently more than 80 JDF-U. Thus, the determination of CFICA is advantageous only when ICA are less than 80 JDF-U.

At the end of follow-up, diabetes had occurred in 11 patients with persistent ICA, in three who seroconverted for ICA during the observation period, and in three without ICA. However, one of the ICA-negative individuals was found to be positive at the onset of the disease. This patient might have been previously identified as positive if shorter antibody detection intervals had been performed.

It has recently been demonstrated that conventional ICA are heterogeneous, with at least two subsets which exhibit different patterns by standard indirect immunofluorescence [22]. One, known as whole or non-restricted, homogeneously stains all endocrine cells of pancreatic islets, while the other, known as selective or restricted, stains only beta cells. The target antigen of the whole ICA pattern has not yet been identified. By contrast, the selective pattern is due to an anti-glutamic acid decarboxylase (GAD) antibody, which is completely abolished by preincubation with tissues rich in GAD [22]. Preliminary observations indicate that the whole pattern is more strongly related to the onset of diabetes both in FDR [23] and in OSAD patients [22]. In the present study we demonstrated that the whole ICA pattern significantly increases the probability of developing diabetes with respect to the selective pattern. Thus, the detection of the whole pattern appears to be the immunological marker which confers the highest risk of progression to diabetes in OSAD patients. In addition, the whole pattern was demonstrated in most patients who developed Type 1 diabetes acutely $(83 \%)$, while it was found in $50 \%$ of those in whom the progression to Type 1 diabetes was slow, i.e. preceded by a transient non-insulin-requiring stage. These data suggest that the identification of the whole or the selective pattern in ICA-positive patients provides further important information not only about the magnitude of the risk but also on the clinical presentation of the diabetic state.

In this cohort of OSAD patients, the prevalence of DR3 and/or DR4 was significantly increased with respect to the general population, and this could account for the lack of correlation found between these haplotypes and susceptibility to diabetes. Interestingly enough, patients with the whole ICA pattern have an increased prevalence of DR3 and/or DR4 when compared to those with the selective pattern, but these differences were not statistically significant, probably due to the small sample of patients available. In this regard, it has been recently reported that in FDR the presence of the restricted ICA pattern (which confers a protective effect) is related to DR2 haplotype [23]. On the basis of these data, it might be speculated that the production of specific ICA subsets are under genetic control also in OSAD patients.

The present study reveals many differences in the natural history of Type 1 diabetes between OSAD patients and FDR with ICA. All our OSAD patients who developed diabetes were females, the mean age at onset was 40 years, and the presentation of Type 1 diabetes was often slow, preceded by a variable period of non-insulin dependence. By contrast, in FDR the disease afflicts both males and females, and has an acute onset between ages $0-20$ years. 
In conclusion, we found that in OSAD patients the whole pattern of ICA is a stronger indicator for future development of overt diabetes than any serological marker which has been investigated, thus confirming similar findings reported in other susceptible populations. The definition of more reliable predictors for Type 1 diabetes represents an essential prerequisite in the selection of those individuals at a very high risk for progressive beta-cell failure and, hopefully, will have a considerable influence on the management of the prediabetic period.

Acknowledgements. We are grateful to Dr. P. Belluardo (Department of Statistical Sciences, University of Florence) for her expert assistance in statistical analysis. The authors acknowledge all the patients involved in this study.

\section{References}

1. Eisenbarth GS (1986) Type 1 diabetes mellitus: a chronic autoimmune disease. N Engl J Med 314: 1360-1368

2. Bottazzo GF, Bonifacio E, Wagner R, Al-Sakkaf L, Dean BM, Mirakian R (1990) On the pathogenesis of type 1 (insulin-dependent) diabetes mellitus: facts, areas still under development and new perspectives. Klin Wochenschr 68 [Suppl $\mathrm{XXI}]: 26-37$

3. Drell DW, Notkins AL (1987) Multiple immunological abnormalities in patients with type 1 (insulin-dependent) diabetes mellitus. Diabetologia 30: 132-143

4. Betterle C, Zanette F, Presotto F, Rapp LB, Monciotti CM, Rigon F (1984) Clinical and subclinical organ-specific autoimmune manifestations in type 1 (insulin-dependent) diabetic patients and their first-degree relatives. Diabetologia 26: $431-436$

5. Betterle C, Callegari G, Presotto Fet al. (1987) Thyroid autoantibodies: a good marker for the study of symptomless autoimmune thyroiditis. Acta Endocrinol (Copenh) 114:321-327

6. Betterle C, Scalici C, Presotto F et al. (1988) The natural history of adrenal function in autoimmune patients with adrenal autoantibodies. J Endocrinol 117; 467-475

7. Bottazzo GF, Genovese S, Bosi E, Dean B, Christie MR, Bonifacio E (1991) Novel considerations on the antibody/autoantigen system in type 1 (insulin-dependent) diabetes mellitus. Ann Med 23: 453-461

8. Bottazzo GF, Florin-Christensen A, Doniach D (1974) Islet cell antibodies in diabetes mellitus with autoimmune polyendocrine deficiencies. Lancet II: 1279-1282

9. Bonifacio E, Lernmark $\AA$, Dawkins RL et al. (1988) Serum exchange and use of dilutions have improved precision of measurement of islet cell antibodies. J Immunol Methods 106: 83-88

10. Bonifacio E, Boitard C, Gleichmann H et al. (1990) Assessment of precision, concordance, specificity, and sensitivity of islet cell antibody measurement in 41 assays. Diabetologia 33: 731-736

11. Srikanta S, Ganda OP, Gleason RE et al. (1983) Type 1 diabetes mellitus in monozygotic twins: chronic progressive B-cell dysfunction. Ann Intern Med 99: 320-326
12. Johnston C, Millward BA, Hoskins P, Leslie RDG, Bottazzo GF, Pyke DA (1989) Islet-cell antibodies as predictors of the later development of type 1 (insulin-dependent) diabetes. A study in identical twins. Diabetologia 32: 382-386

13. Tarn AC, Thomas JM, Dean BM et al. (1988) Predicting insulin-dependent diabetes. Lancet I: 845-850

14. Ziegler AG, Ziegler R, Vardi P, Jackson RA, Soeldner JS, Eisenbarth G (1989) Life-table analysis of progression to diabetes of anti-insulin autoantibody-positive relatives of individuals with type 1 diabetes. Diabetes 38: 1320-1325

15. Riley WJ, Maclaren NK, Krischer J et al. (1990) A prospective study of the development of diabetes in relatives of patients with insulin-dependent diabetes. N Engl J Med 323: 1167-1172

16. Deschamps I, Boitard C, Hors J et al. (1992) Life table analysis of the risk of type 1 (insulin-dependent) diabetes mellitus in siblings according to islet cell antibodies and HLA markers. An 8-year prospective study. Diabetologia 35: 951-957

17. Riley WJ, Winter WE, Maclaren NK (1988) Identification of insulin-dependent diabetes mellitus before the onset of clinical symptoms. J Pediatr 112: 314-316

18. Bruining GJ, Molenaar JL, Grobbee DE et al. (1989) Tenyear follow-up study of islet-cell antibodies and childhood diabetes mellitus. Lancet I: 1100-1103

19. Karjalainen JK (1990) Islet cell antibodies as predictive markers for IDDM in children with high background incidence of disease. Diabetes 39: 1144-1150

20. Betterle C, Presotto F, Pedini B et al. (1987) Islet cell and insulin autoantibodies in organ-specific autoimmune patients. Their behaviour and predictive value in the development of type 1 (insulin-dependent) diabetes mellitus. A ten year follow-up study. Diabetologia 30: 292-297

21. Bosi E, Becker F, Bonifacio E et al. (1991) Progression to type 1 diabetes in autoimmune endocrine patients with islet cell antibodies. Diabetes 40: 977-984

22. Genovese S, Bonifacio E, McNally JM et al. (1992) Distinct cytoplasmic islet cell antibodies with different risks for type 1 (insulin-dependent) diabetes mellitus. Diabetologia 35: 385-388

23. Gianani R, Pugliese A, Bonner-Weir S et al. (1992) Prognostically significant heterogeneity of cytoplasmic islet cell antibodies in relatives of patients with type 1 diabetes. Diabetes 41:347-353

24. National Diabetes Data Group (1979) Classification and diagnosis of diabetes mellitus and other categories of glucose intolerance. Diabetes 28: 1039-1057

25. Cutler SJ, Ederer F (1958) Maximum utilization of the life table in analyzing survival. J Chron Dis 8: 699-712

26. Peto R, Pike MC, Armitage P et al. (1977) Design and analysis of randomised clinical trials requiring prolonged observation of each patient. II Analysis and examples. Br J Cancer 35: $1-39$

27. Genovese S, Wagner R, Bonifacio E et al. (1992) Absence of immune abnormalities in the pancreases of non-diabetic patients with long-standing GAD-antibodies. Diabetologia 35 [Suppl 1]: A41 (Abstract)

28. Bonifacio E, Bingley PJ, Shattock Met al. (1990) Quantification of islet-cell antibodies and prediction of insulin-dependent diabetes. Lancet I: 147-149 